\title{
Investing in a Random Start American Option Under Competition周
}

\author{
Paulo J. Pereira $a^{\ddagger}$ and Artur Rodrigues ${ }^{\S}$ \\ ${ }^{\ddagger}$ CEF. UP and Faculdade de Economia, Universidade do Porto. \\ $\S$ NIPE and School of Economics and Management, University of Minho.
}

June 2018

\begin{abstract}
In this paper we develop a model to determine the value of the opportunity to invest in a random start American real option. In contrast to a typical American option, the random start option only exists if an exogenous event occurs materializing the American option to invest. In addition, the effect of competition is also considered in the model. A higher risk of competition and a higher probability of the exogenous event promotes investment. Uncertainty has a non-monotonic effect on investment timing.
\end{abstract}

Keywords: Random start options; Real options; Uncertainty; Competition.

JEL codes: G13; D81.

${ }^{*}$ This work was carried out within the funding with COMPETE reference n. POCI-01-0145-FEDER006683 (Artur Rodrigues) and POCI-01-0145-FEDER-006890 (Paulo J. Pereira), FCT/MEC's (Fundação para a Ciência e a Tecnologia, I.P.) financial support through national funding and by ERDF through the Operational Programme on Competitiveness and Internationalization - COMPETE 2020 under the PT2020 Partnership Agreement. 


\section{Investing in a Random Start American Option Under Competition}

\section{Introduction}

In this paper we develop a model capable of determining the value of the opportunity to invest in a random start American real option. In contrast to a typical American option, the random start option (RSO) ${ }_{1}^{1}$ only exists if some exogenous event occurs. The random (exogenous) event is assumed to be outside of the investor's control, and only after it occurs the (true) American option to invest materializes.

Several examples fit with this setting. An investment opportunity that depends on the authorization of a public entity, which may eventually arrive in the future (e.g., the license to transform a rural land, with construction limitations, into an urban one); an R\&D race where the discovery arrives randomly (Lint and Pennings, 1998); or a project that depends on a technology developed by a third-party firm (e.g., the iPad was dependent on an efficient multi-touch screen technology, developed outside Apple).

In the context of our examples, the initial capital investment could correspond to the acquisition of the rural land with the expectation that it will be later transformed into urban by the authorities (by acquiring the land the investor becomes proprietary of the random start option). Similarly, the firm can invest in patenting the potential discovery that may randomly arrive during the $\mathrm{R} \& \mathrm{D}$ process (the alternative that does not eliminate competition would be to patent the discover only if and when it occurs). Finally, for the last example, the firm can pay a third-party to secure exclusive rights in the case the technology arrives, ensuring monopolistic rents.

Our paper closely relates to Armerin (2017). The author also considers a similar American option that can only be exercised after a random period of time has passed. We differ in two major ways. Firstly, in contrast to Armerin's work, that considers that the firm already owns the random start American option, we go one step back and consider the decision to acquire the RSO. In other words, we depart from the assumption that the firm is, ex ante, endowed with the random start option, modeling, instead, the decision to acquire it. Secondly, we consider that the firm has no proprietary rights on the RSO, incorporating competition for the acquisition of the option.

The paper unfolds as follows. Section 2 develops the model for investing in a random start American options under competition. Section 3 presents a numerical example with a comparative statics, highlighting the main insights of the model. Section 4 concludes.

\footnotetext{
${ }^{1}$ We use interchangeably "random start American option", "random start option", or simply RSO.
} 


\section{The Model}

Consider a real asset that produces a stream of cash flows. The present value of these cash flows, $X(t)$, is assumed to follow a geometric Brownian motion:

$$
d X(t)=\alpha X(t) d t+\sigma X(t) d B(t)
$$

where $X(0)=X>0, \alpha<r$ is the risk-neutral expected drift, $r$ is the risk-free rate, $\sigma$ the instantaneous volatility, and $d B$ is the increment of a Wiener process.

The investment in this project has two stages. The first stage, in which $K_{1}$ is invested, allows the firm to become a monopolist over the second stage of the project, eliminating any possible competitive damage. However, the investment in this second stage, depends on some exogenous event without which the project is noneffective. After this event, the firm is entitled with a perpetual American option to invest, which requires a lump sum investment of $K_{2}$. However, notice that if the exogenous event happens to occur before the firm invests $K_{1}$ (i.e., before securing monopolistic rights over the second stage), the option to invest in the project is shared with competitors.

This model considers three types of uncertainties. Firstly, the cash flows of the project evolve randomly over time. Secondly, the effectiveness of the project depends on some exogenous event. Lastly, competition is also considered by including the existence of hidden rivals (Armada et al., 2011; Pereira and Armada, 2013, Lavrutich et al., 2016).

Figure 1 exhibits all possible states. In the beginning, the firm holds $F(X)$. This is a non-proprietary option to invest $K_{1}$ and receive $G(X)$, becoming a monopolist over the next stage. Two possible events may occur while the firm holds $F(X)$ : the exogenous event occurs (transforming $F(X)$ into $H_{C}(X)$ ) or a (hidden) competitor moves in and invests $K_{1}$, and $F(X)$ becomes worthless for the company. After investing $K_{1}$ the firm is entitled with the monopolistic option $G(X)$. This option ends-up to be $H(X)$ if the exogenous event occurs. $H(X)$ is the perpetual American option to invest $K_{2}$ and receive $X$. Additionally, if the exogenous event occurs before the firm makes the first investment (before investing $\left.K_{1}\right), F(X)$ is transformed into $H_{C}(X)$, which corresponds to the non-monopolistic option to invest in the second stage. Given that $H_{C}(X)$ can suddenly disappear if a competitor preempts the firm, $K_{1}$ can be paid in order to secure the position of monopolist of the project $(H(X))$.

For solving the model we proceed backwards, starting with the last option $H(X)$, and then moving to the earlier stages.

\subsection{The value of the project after the exogenous event}

After the exogenous event that allows the firm to invest in the last stage, the firm can either have secured the monopolistic option to invest (by having invested $K_{1}$ ) or is still 


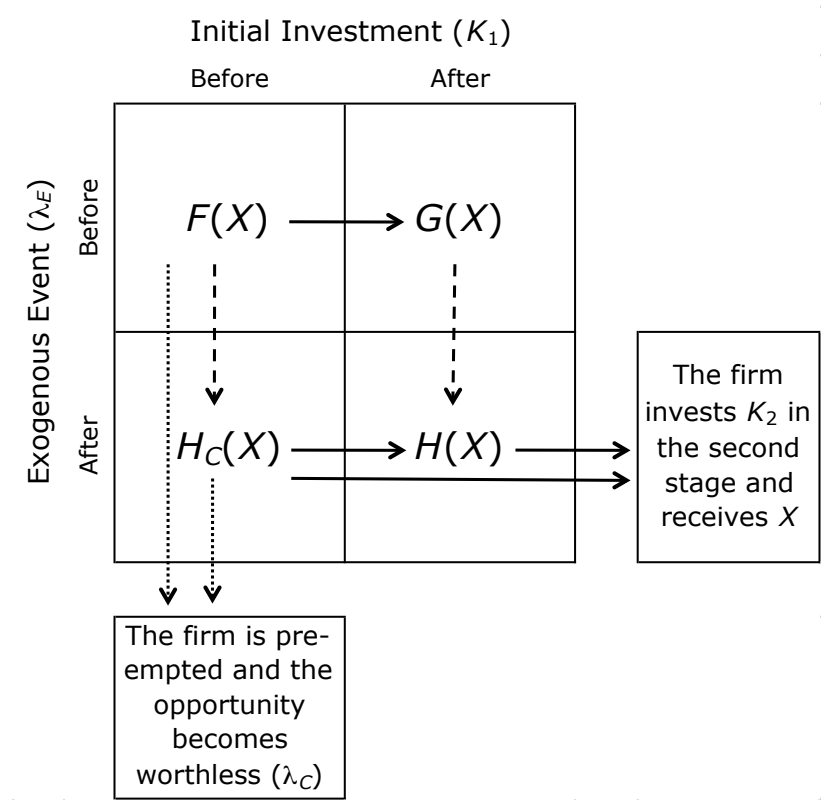

Figure 1: The solid lines represent the changes in the value functions as a result of firm's decisions (first stage and second stage investment). The dashed and the dotted lines represent, respectively, the change in the value functions if the exogenous event occurs or if the firm is preempted by a competitor.

waiting to secure the investment and faces the hidden competition.

\subsubsection{The monopolistic right to invest in the last stage}

Let $H(X)$ be the value of the proprietary option to invest in the last stage, under which the firm receives $X$ in exchange for the sunk investment cost $K_{2}$. Following the standard procedures, $H(X)$ is the solution to the following ordinary differential equation (ODE):

$$
\frac{1}{2} \sigma^{2} X^{2} H^{\prime \prime}(X)+\alpha X H^{\prime}(X)-r H(X)=0
$$

The solution is the well known option to invest value (McDonald and Siegel, 1986; Dixit and Pindyck, 1994):

$$
H(X)= \begin{cases}a_{1} X^{\beta_{1}} & \text { for } X<X_{2} \\ X-K_{2} & \text { for } X \geqslant X_{2}\end{cases}
$$

where

$$
a_{1}=\left(X_{2}-K_{2}\right)\left(\frac{1}{X_{2}}\right)^{\beta_{1}}=\frac{K_{2}}{\beta_{1}-1}\left(\frac{1}{X_{2}}\right)^{\beta_{1}}
$$




$$
\beta_{1}=\frac{1}{2}-\frac{\alpha}{\sigma^{2}}+\sqrt{\left(-\frac{1}{2}+\frac{\alpha}{\sigma^{2}}\right)^{2}+\frac{2 r}{\sigma^{2}}}
$$

and $X_{2}$ is the threshold for investment:

$$
X_{2}=\frac{\beta_{1}}{\beta_{1}-1} K_{2}
$$

\subsubsection{The shared option to invest in the last stage}

Let $H_{C}(X)$ be the value function of the option to invest when the firm may be preempted by a hidden competitor, destroying the option value. That event is modeled as a Poisson event with intensity $\lambda_{C} . H_{C}(X)$ is the solution to the following ODE (Dixit and Pindyck, 1994):

$$
\frac{1}{2} \sigma^{2} X^{2} H_{C}^{\prime \prime}(X)+\alpha X H_{C}^{\prime}(X)-r H_{C}(X)+\lambda_{C}\left(0-H_{C}(X)\right)=0
$$

and, considering the boundary when $X \rightarrow 0$, is given by:

$$
H_{C}(X)=b X^{\eta_{1}}
$$

where

$$
\eta_{1}=\frac{1}{2}-\frac{\alpha}{\sigma^{2}}+\sqrt{\left(-\frac{1}{2}+\frac{\alpha}{\sigma^{2}}\right)^{2}+\frac{2\left(r+\lambda_{C}\right)}{\sigma^{2}}}
$$

The firm can choose between two alternative strategies to kill competition: (1) to stage the investment, investing $K_{1}$ to secure a monopolistic position over the project, or (2) invest immediately in the two stages $\left(K_{1}+K_{2}\right)$. The optimal strategy will be the most valuable and not necessarily that with the earliest threshold.

\section{Case 1: Staged investment}

Under this strategy the firm will choose to secure the option to invest in the last stage by paying $K_{1}$ in the first stage and not pre-committing to the second stage investment. Doing so, the firm acquires the exclusive option to invest $H(X)$. It only makes economic sense to stage the investment if the threshold of the second stage $X_{2}$ has bot been reached. Therefore, the value-matching and smooth-pasting boundary conditions, at the threshold $X_{11}^{c}<X_{2}$, are:

$$
\begin{aligned}
b_{1} X_{11}^{c} \eta_{1} & =a_{1} X_{11}^{c \beta_{1}}-K_{1} \\
\eta_{1} b_{1} X_{11}^{c} \eta_{1}-1 & =\beta_{1} a_{1} X_{11}^{c} \beta_{1}-1
\end{aligned}
$$


These boundary conditions produce the following solution for the option value:

$$
H_{C}(X)= \begin{cases}b_{1} X^{\eta_{1}} & \text { for } X<X_{11}^{c} \\ a_{1} X^{\beta_{1}}-K_{1} & \text { for } X_{11}^{c} \leqslant X<X_{2} \\ X-K_{2}-K_{1} & \text { for } X \geqslant X_{2}\end{cases}
$$

where

$$
b_{1}=\left(a_{1} X_{11}^{c} \beta_{1}-K_{1}\right)\left(\frac{1}{X_{11}^{c}}\right)^{\eta_{1}}=\frac{\beta_{1} K_{1}}{\eta_{1}-\beta_{1}}\left(\frac{1}{X_{11}^{c}}\right)^{\eta_{1}}
$$

and $X_{11}^{c}$ is the threshold:

$$
X_{11}^{c}=X_{2}\left(\frac{\eta_{1}\left(\beta_{1}-1\right)}{\eta_{1}-\beta_{1}} \frac{K_{1}}{K_{2}}\right)^{\frac{1}{\beta_{1}}}
$$

The condition that the threshold $X_{2}$ must be greater that $X_{11}^{c}$ implies that the initial investment $K_{1}$ must be sufficiently smaller that $K_{2}$ :

$$
K_{1}<\frac{\eta_{1}-\beta_{1}}{\eta_{1}\left(\beta_{1}-1\right)} K_{2}
$$

For the limiting cases where competition is absent $\left(\lambda_{C}=0\right)$ or is imminent $\left(\lambda_{C} \rightarrow \infty\right)$, the condition becomes $K_{1}<0$ and $K_{1}<K_{2} /\left(\beta_{1}-1\right)$, respectively. When there are no potential competitors, staging the investment is excluded because the firm holds an exclusive option on the second stage investment, while when the competitor is about to make the investment, the firm can pay the maximum amount $K_{2} /\left(\beta_{1}-1\right)$ to secure the investment. A higher risk of competition (higher $\lambda_{C}$ or equivalently a higher $\eta_{1}$ ) induces the firm to be available to pay a larger $K_{1}$. Notice that the higher the market uncertainty (lower $\beta_{1}$ ), the larger the amount a firm is willing to pay to secure the exclusive right to later invest in the second stage.

\section{Case 2: Investment in a single stage}

Under this strategy the firm will choose the two investments $\left(K_{1}+K_{2}\right)$ in a single stage, eliminating competition.

The following value-matching and smooth-pasting boundary conditions, at the threshold $X_{12}^{c}$ :

$$
\begin{aligned}
b X_{12}^{c} \eta_{1} & =X-\left(K_{1}+K_{2}\right) \\
\eta_{1} b X_{12}^{c \eta_{1}-1} & =1
\end{aligned}
$$


produce the following solution:

$$
H_{C}(X)= \begin{cases}b_{2} X^{\eta_{1}} & \text { for } X<X_{12}^{c} \\ X-\left(K_{1}+K_{2}\right) & \text { for } X \geqslant X_{12}^{c}\end{cases}
$$

where

$$
b_{2}=\left(X_{12}^{c}-\left(K_{1}+K_{2}\right)\right)\left(\frac{1}{X_{12}^{c}}\right)^{\eta_{1}}=\frac{K_{1}+K_{2}}{\eta_{1}-1}\left(\frac{1}{X_{12}^{c}}\right)^{\eta_{1}}
$$

and $X_{12}^{c}$ is the threshold:

$$
X_{12}^{c}=\frac{\eta_{1}}{\eta_{1}-1}\left(K_{1}+K_{2}\right)
$$

A higher risk of competition (higher $\lambda_{C}$ or higher $\left.\eta_{1}\right)$ hastens investment $\left(\partial X_{12}^{c} / \partial \lambda_{C}<\right.$ 0 ). On the other hand, a higher market uncertainty (lower $\eta_{1}$ ) deters investment ${ }^{2}$

\section{Optimal strategy}

A firm will prefer to stage the investment if the value of that strategy is higher than that of the alternative single stage investment $\left(b_{1}>b_{2}\right)$, even if the threshold of the latter $\left(X_{12}^{c}\right)$ is reached before the threshold of the former $\left(X_{11}^{c}\right)$. The following condition must hold for a staged investment to be preferred:

$$
\left(\frac{X_{12}^{c}}{X_{11}^{c}}\right)^{\eta_{1}}>\frac{\eta_{1}-\beta_{1}}{\beta_{1}\left(\eta_{1}-1\right)}\left(\frac{K_{1}+K_{2}}{K_{1}}\right)
$$

It is possible to prove that this condition always holds. 3 Therefore, we need only condition (15) to define the optimal strategy.

\subsection{The value of the project before the exogenous event}

Before the exogenous event that allows the firm to invest in the last stage, the firm can choose between securing the monopolistic option to invest or waiting and sharing the option with hidden competitors. Let us assume that securing the investment before the exogenous event can be less costly, i.e. the investment cost is $\theta K_{1}(0<\theta \leqslant 1)$.

\subsubsection{The value of the monopolistic option invest in the first stage}

After paying $\theta K_{1}$, the firm secures the investment opportunity $H(X)$ killing competition and waits for the occurrence of the exogenous event that permits the investment in the last stage. This event arrives according to a Poisson process with an intensity rate $\lambda_{E}$. Let $G(X)$ be the value of the monopolistic option, which must be the solution to the following

\footnotetext{
${ }^{2}$ Notice that $\partial \eta_{1} / \partial \sigma<0$.

${ }^{3}$ Proof available upon request.
} 
ODE:

$$
\frac{1}{2} \sigma^{2} X^{2} G^{\prime \prime}(X)+\alpha X G^{\prime}(X)-r G(X)+\lambda_{E}(H(X)-G(X))=0
$$

The exogenous event can occur either before or after the threshold $X_{2}$ has been reached. The two regions of $H(X)$ shown in Equation (18) produce the following solution to the above ODEA

$$
G(X)= \begin{cases}c_{1} X^{\gamma_{1}}+a_{1} X^{\beta_{1}} & \text { for } X<X_{2} \\ c_{4} X^{\gamma_{2}}+\Lambda_{1} X-\Lambda_{2} K_{2} & \text { for } X \geqslant X_{2}\end{cases}
$$

where

$$
\begin{aligned}
\Lambda_{1} & =\frac{\lambda_{E}}{r-\alpha+\lambda_{E}} \\
\Lambda_{2} & =\frac{\lambda_{E}}{r+\lambda_{E}} \\
\gamma_{1} & =\frac{1}{2}-\frac{\alpha}{\sigma^{2}}+\sqrt{\left(-\frac{1}{2}+\frac{\alpha}{\sigma^{2}}\right)^{2}+\frac{2\left(r+\lambda_{E}\right)}{\sigma^{2}}} \\
\gamma_{2} & =\frac{1}{2}-\frac{\alpha}{\sigma^{2}}-\sqrt{\left(-\frac{1}{2}+\frac{\alpha}{\sigma^{2}}\right)^{2}+\frac{2\left(r+\lambda_{E}\right)}{\sigma^{2}}}
\end{aligned}
$$

and $a_{1}$ and $X_{2}$ are as in Equations (4) and (6), respectively, and the constants $a_{3}$ and $a_{4}$ ensure that $G(X)$ is continuous and differentiable along $X[5$

$$
\begin{aligned}
& c_{1}=\frac{\left(\beta_{1}-\gamma_{2}\right)\left(X_{2}-K_{2}\right)+\Lambda_{1}\left(\gamma_{2}-1\right) X_{2}-\Lambda_{2} \gamma_{2} K_{2}}{\gamma_{2}-\gamma_{1}}\left(\frac{1}{X_{2}}\right)^{\gamma_{1}} \\
& c_{4}=\frac{\left(\beta_{1}-\gamma_{1}\right)\left(X_{2}-K_{2}\right)+\Lambda_{1}\left(\gamma_{1}-1\right) X_{2}-\Lambda_{2} \gamma_{1} K_{2}}{\gamma_{2}-\gamma_{1}}\left(\frac{1}{X_{2}}\right)^{\gamma_{2}}
\end{aligned}
$$

\subsubsection{The value of a random start American real option under competition}

Let $F(X)$ be the value of a random start American real option under competition, i.e. the value of the shared option to invest in the first stage, whose value must be the solution to the following ODE:

$$
\frac{1}{2} \sigma^{2} X^{2} F^{\prime \prime}(X)+\alpha X F^{\prime}(X)-r F(X)+\lambda_{E}\left(H_{C}(X)-F(X)\right)+\lambda_{C}(0-F(X))=0
$$

where $\lambda_{E}$ is the arrival rate of the exogenous event, and $\lambda_{C}$ corresponds to the arrival rate of a competitor that preempts the firm, killing the option value.

Depending on condition (15), $H_{C}(X)$ is given by Equation $(12)$ or Equation (18), each

\footnotetext{
${ }^{4}$ After considering the boundary condition when $X \rightarrow 0$ and $X \rightarrow \infty$. This option corresponds to the case presented in Armerin (2017). We use a different solution strategy that produces a different analytical solution. Our numerical simulations have shown that the option values are exactly the same.

${ }^{5}$ Using the value-matching and smooth-pasting conditions at $X_{2}$.
} 
of them with more than one branch.

Let $X_{1}$ be the threshold for investment in the first stage. The following cases emerge:

Table 1: Investment strategy cases

\begin{tabular}{|c|c|c|c|c|}
\hline & \multicolumn{2}{|c|}{ 1. Staged investment } & \multicolumn{2}{c|}{ 2. Single stage investment } \\
& $X_{1}<X_{11}^{c}$ & $X_{1} \geqslant X_{11}^{c}$ & $X_{1}<X_{12}^{c}$ & $X_{1} \geqslant X_{12}^{c}$ \\
\hline$X_{1}<X_{2}$ & $\mathrm{~A}$ & $\mathrm{C}$ & $\mathrm{E}$ & $\mathrm{G}$ \\
\hline$X_{1} \geqslant X_{2}$ & $\mathrm{~B}$ & $\mathrm{D}$ & $\mathrm{F}$ & $\mathrm{H}$ \\
\hline
\end{tabular}

Staged investment occurs when condition in Equation 15 is met. The four cases for each investment strategy arise depending on the model parameters.

The solution for the threshold, $X_{1}$, and the option value, $F(X)$, is obtained using the value-matching and smooth-pasting conditions:

$$
\begin{aligned}
& F\left(X_{1}\right)=G\left(X_{1}\right)-\theta K_{1} \\
& \left.\frac{\partial F(X)}{\partial X}\right|_{X=X_{1}}=\left.\frac{\partial G(X)}{\partial X}\right|_{X=X_{1}}
\end{aligned}
$$

\section{Case 1: Staged investment}

Considering the boundary condition when $X \rightarrow 0$, the solution to the ODE (30) has three branches:

$$
F(X)= \begin{cases}d_{1} X^{\psi_{1}}+b_{1} X^{\eta_{1}} & \text { for } X<X_{11}^{c} \\ d_{3} X^{\psi_{1}}+d_{4} X^{\psi_{2}}+\Lambda_{4} a_{1} X^{\beta_{1}}-\Lambda_{3} K_{1} & \text { for } X_{11}^{c} \leqslant X<X_{2} \\ d_{5} X^{\psi_{1}}+d_{6} X^{\psi_{2}}+\Lambda_{1}\left(X-K_{2}-K_{1}\right) & \text { for } X \geqslant X_{2}\end{cases}
$$

where

$$
\begin{aligned}
& \Lambda_{3}=\frac{\lambda_{E}}{r+\lambda_{C}+\lambda_{E}} \\
& \Lambda_{4}=\frac{\lambda_{E}}{\lambda_{C}+\lambda_{E}} \\
& \psi_{1}=\frac{1}{2}-\frac{\alpha}{\sigma^{2}}+\sqrt{\left(-\frac{1}{2}+\frac{\alpha}{\sigma^{2}}\right)^{2}+\frac{2\left(r+\lambda_{E}+\lambda_{C}\right)}{\sigma^{2}}} \\
& \psi_{2}=\frac{1}{2}-\frac{\alpha}{\sigma^{2}}-\sqrt{\left(-\frac{1}{2}+\frac{\alpha}{\sigma^{2}}\right)^{2}+\frac{2\left(r+\lambda_{E}+\lambda_{C}\right)}{\sigma^{2}}}
\end{aligned}
$$


Cases A and B The value for the investment opportunity is:

$$
F(X)= \begin{cases}d_{11} X^{\psi_{1}}+b_{1} X^{\eta_{1}} & \text { for } X<X_{1} \\ G(X)-\theta K_{1} & \text { for } X \geqslant X_{1}\end{cases}
$$

where

$$
d_{11}=\left(G\left(X_{1}\right)-\theta K_{1}-b_{1} X_{1}^{\eta_{1}}\right)\left(\frac{1}{X_{1}}\right)^{\psi_{1}}
$$

and the trigger, $X_{1}$, is numerically obtained by solving the following equations:

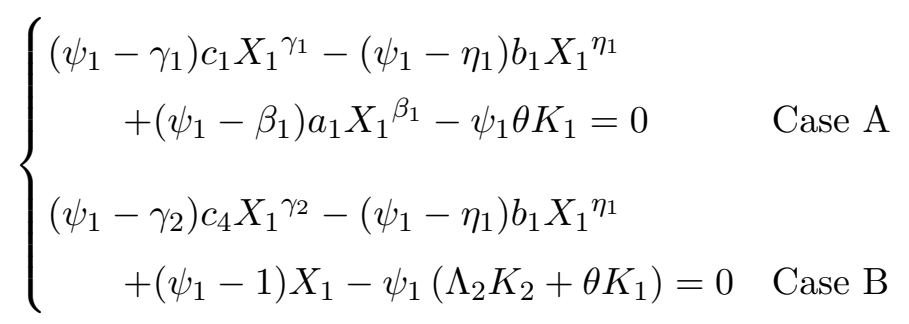

Cases C and D The value for the investment opportunity is:

$$
F(X)= \begin{cases}d_{13} X^{\psi_{1}}+b_{1} X^{\eta_{1}} & \text { for } X<X_{11}^{c} \\ L(X) & \text { for } X_{11}^{c} \leqslant X<X_{1} \\ G(X)-\theta K_{1} & \text { for } X \geqslant X_{1}\end{cases}
$$

where

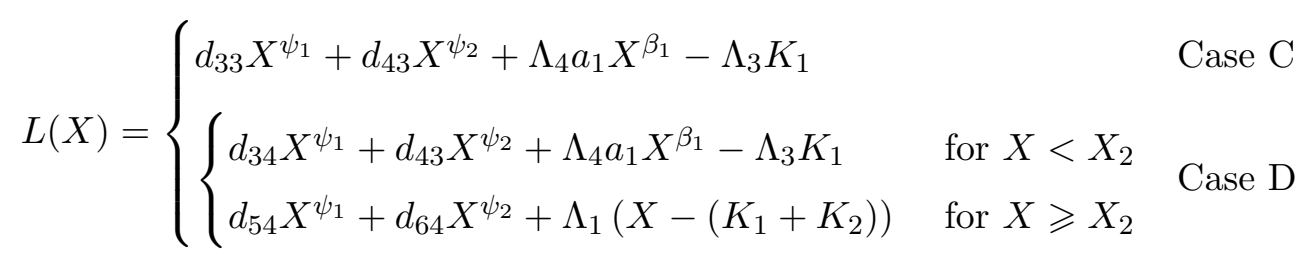




$$
\begin{aligned}
& d_{13}=\left(d_{43} X_{11}^{c} \psi_{2}-\left(1-\Lambda_{4}\right) a_{1} X_{11}^{c} \beta_{1}+\left(1-\Lambda_{3}\right) K_{1}\right)\left(\frac{1}{X_{11}^{c}}\right)^{\psi_{1}}+ \begin{cases}d_{33} & \text { Case C } \\
d_{34} & \text { Case D }\end{cases} \\
& d_{33}=\left(c_{1} X_{1}{ }^{\gamma_{1}}+\left(1-\Lambda_{4}\right) a_{1} X_{1}{ }^{\beta_{1}}-\left(\theta-\Lambda_{3}\right) K_{1}-d_{43} X_{1} \psi_{2}\right)\left(\frac{1}{X_{1}}\right)^{\psi_{1}} \\
& d_{34}=d_{54}+\left(\left(d_{64}-d_{43}\right) X_{2}{ }^{\psi_{2}}-\left(\Lambda_{4}-\Lambda_{3}\right)\left(X_{2}-K_{2}\right)\right)\left(\frac{1}{X_{2}}\right)^{\psi_{1}} \\
& d_{43}=\frac{\left(\psi_{1}-\beta_{1}\right)\left(1-\Lambda_{4}\right) a_{1} X_{11}^{c} \beta_{1}-\psi_{1}\left(1-\Lambda_{3}\right) K_{1}}{\psi_{1}-\psi_{2}}\left(\frac{1}{X_{11}^{c}}\right)^{\psi_{2}} \\
& d_{54}=\left(c_{4} X_{1} \gamma_{2}+\left(\Lambda_{1}-\Lambda_{3}\right) X_{1}-\left(\Lambda_{2}-\Lambda_{3}\right) K_{2}-\left(\theta-\Lambda_{3}\right) K_{1}-d_{64} X_{1} \psi_{2}\right)\left(\frac{1}{X_{1}}\right)^{\psi_{1}} \\
& d_{64}=d_{43}+\left(\Lambda_{4}-\Lambda_{3}\right) \frac{\left(\psi_{1}-1\right) X_{2}-\psi_{1} K_{2}}{\psi_{1}-\psi_{2}}\left(\frac{1}{X_{2}}\right)^{\psi_{2}}
\end{aligned}
$$

and the trigger, $X_{1}$, is numerically obtained by solving the following equations:

$$
\begin{cases}-\left(\psi_{1}-\psi_{2}\right) d_{43} X_{1} \psi_{2}+\left(\psi_{1}-\gamma_{1}\right) c_{1} X_{1}{ }^{\gamma_{1}}+\left(\psi_{1}-\beta_{1}\right)\left(1-\Lambda_{4}\right) a_{1} X_{1} \beta_{1} & \\ -\psi_{1}\left(\theta-\Lambda_{3}\right) K_{1}=0 & \text { Case C } \\ -\left(\psi_{1}-\psi_{2}\right) d_{64} X_{1} \psi_{2}+\left(\psi_{1}-\gamma_{2}\right) c_{4} X_{1}{ }^{\gamma_{2}}+\left(\psi_{1}-1\right)\left(\Lambda_{1}-\Lambda_{3}\right) X_{1} & \\ -\psi_{1}\left(\left(\Lambda_{2}-\Lambda_{3}\right) K_{2}+\left(\theta-\Lambda_{3}\right) K_{1}\right)=0 & \text { Case D }\end{cases}
$$

\section{Case 2: Single stage investment}

For the case of a single stage investment, Equation 18 is used to find the solution to the ODE (30). The solution with two branches, considering the boundary condition when $X \rightarrow 0$, is the following:

$$
F(X)= \begin{cases}e_{1} X^{\psi_{1}}+b_{2} X^{\eta_{1}} & \text { for } X<X_{12}^{c} \\ e_{3} X^{\psi_{1}}+e_{4} X^{\psi_{2}}+\Lambda_{3}\left(X-\left(K_{1}+K_{2}\right)\right) & \text { for } X \geqslant X_{12}^{c}\end{cases}
$$

Cases $\mathbf{E}$ and $\mathbf{F}$ The value of the investment opportunity is:

$$
F(X)= \begin{cases}e_{11} X^{\psi_{1}}+b_{2} X^{\eta_{1}} & \text { for } X<X_{1} \\ G(X)-\theta K_{1} & \text { for } X \geqslant X_{1}\end{cases}
$$

where

$$
e_{11}=\left(G\left(X_{1}\right)-\theta K_{1}-b_{2} X_{1}^{\eta_{1}}\right)\left(\frac{1}{X_{1}}\right)^{\psi_{1}}
$$


and the trigger, $X_{1}$, is numerically obtained by solving the following equations:

$$
\begin{cases}\left(\psi_{1}-\gamma_{1}\right) c_{1} X_{1}^{\gamma_{1}}-\left(\psi_{1}-\eta_{1}\right) b_{2} X_{1}{ }^{\eta_{1}}+\left(\psi_{1}-\beta_{1}\right) a_{1} X_{1}^{\beta_{1}}-\psi_{1} \theta K_{1}=0 & \text { Case E } \\ \left(\psi_{1}-\gamma_{2}\right) c_{4} X_{1} \gamma_{2}-\left(\psi_{1}-\eta_{1}\right) b_{2} X_{1}{ }^{\eta_{1}}+\left(\psi_{1}-1\right) X_{1}-\psi_{1}\left(\Lambda_{2} K_{2}+\theta K_{1}\right)=0 & \text { Case F }\end{cases}
$$

Cases $\mathbf{G}$ and $\mathbf{H}$ The value for the investment opportunity is:

$$
F(X)= \begin{cases}e_{13} X^{\psi_{1}}+b_{2} X^{\eta_{1}} & \text { for } X<X_{12}^{c} \\ e_{33} X^{\psi_{1}}+e_{43} X^{\psi_{2}}+\Lambda_{3}\left(X-\left(K_{1}+K_{2}\right)\right) & \text { for } X_{12}^{c} \leqslant X<X_{1} \\ G(X)-\theta K_{1} & \text { for } X \geqslant X_{1}\end{cases}
$$

where

$$
\begin{aligned}
& e_{13}=e_{33}+\left(e_{43} X_{12}^{c} \psi_{2}-\left(1-\Lambda_{3}\right)\left(X_{12}^{c}-\left(K_{1}+K_{2}\right)\right)\right)\left(\frac{1}{X_{12}^{c}}\right)^{\psi_{1}} \\
& e_{33}=\left(G\left(X_{1}\right)-\theta K_{1}-e_{43} X_{1} \psi_{2}-\Lambda_{3}\left(X_{1}-\left(K_{1}+K_{2}\right)\right)\right)\left(\frac{1}{X_{1}}\right)^{\psi_{1}} \\
& e_{43}=\left(1-\Lambda_{3}\right) \frac{\left(\psi_{1}-1\right) X_{12}^{c}-\psi_{1}\left(K_{1}+K_{2}\right)}{\psi_{1}-\psi_{2}}\left(\frac{1}{X_{12}^{c}}\right)^{\psi_{2}}
\end{aligned}
$$

and the trigger, $X_{1}$, is numerically obtained by solving the following equations:

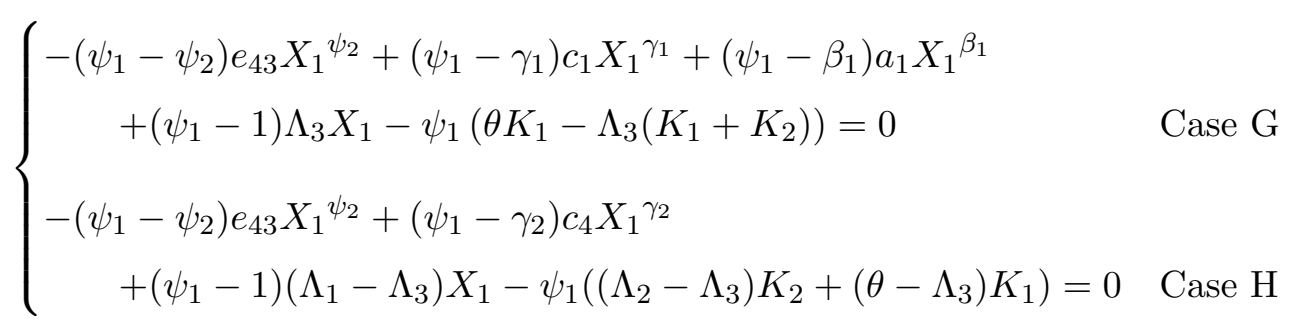

\section{Numerical example and comparative statics}

Let us consider the case of a real estate firm contemplating the acquisition of a piece of rural land that does not have a construction permit. The land development may be allowed in future at an unknown date, here modeled as an Poisson event with an arrival rate of $\lambda_{E}$. Additionally, the investment opportunity is shared with hidden competitors that may preempt the firm (with an arrival rate of $\lambda_{C}$ ). The land acquisition, that eliminates competition, may be optimal prior or after the construction permit being issued. Using a numerical example, we illustrate how the optimal decision is affect by the model parameters, presented in Table 2 .

Table 3 tabulates several numerical examples, including some limiting cases. When 
Table 2: The base case parameters

\begin{tabular}{clc}
\hline Parameter & Description & Value \\
\hline$\sigma$ & Volatility of the cash flows & 0.1 \\
$r$ & Risk-free rate & 0.04 \\
$\alpha$ & Risk-neutral growth rate of the cash flows & 0.02 \\
$K_{1}$ & Cost of the land after the permit (stage 1) & 10 \\
$\theta K_{1}$ & Cost of the land before the permit (stage 1) & 9 \\
$K_{2}$ & Development cost (stage 2) & 50 \\
$\lambda_{E}$ & Arrival rate of the construction permit & 0.05 \\
$\lambda_{C}$ & Arrival rate of a competitor & 0.1 \\
\hline
\end{tabular}

the permit to construct has a zero probability of being issued $\left(\lambda_{E} \rightarrow 0\right)$ investment will never occur $\left(X_{1} \rightarrow \infty\right)$. When the permit is imminent $\left(\lambda_{E} \rightarrow \infty\right)$, investment is hastened the most (the difference between $X_{1}<X_{1}^{c}$ reaches the maximum ${ }^{6}$ For the particular case where both events (the arrival of a competitor and the permit $-\lambda_{E} \rightarrow \infty$ and $\lambda_{C} \rightarrow \infty$ ) are imminent, the firm will invest at the minimum threshold possible, paying $\theta K_{1}$ to secure the exclusive option to invest in the development stage $\left(H(X)=a_{1} X^{\beta_{1}}\right)$ when the payoff is zero $\left(H(X)=\theta K_{1}\right)$.

Figure 2 shows that a higher risk of a competitor arrival $\left(\lambda_{C}\right)$ or a higher likelihood of the construction permit being issued $\left(\lambda_{E}\right)$ induce an earlier investment. When these events have a low probability of occurrence, the investment is delayed and can even occur later than when the land development becomes optimal, if allowed $\left(X_{1}>X_{2}\right)$. The figure also shows that a higher discount (lower $\theta$ ) of acquiring the land before the permit accelerates investment. When we compare the thresholds for the acquisition of the land before and after the permit is issued $\left(X_{1}\right.$ and $\left.X_{1}^{c}\right)$, it is possible to conclude that the level of discount $(\theta)$ determines if the investment in the first stage occurs later or sooner than when it becomes optimal after the permit. In particular, when there is no discount $(\theta=1)$ it is always optimal to invest later if the permit has not been issued $\left(X_{1}>X_{1}^{c}\right)$.

The firm also faces another source of risk - the cash flows risk measured by the volatility parameter $\sigma$. Figure 3 shows an unusual effect of uncertainty. Usually uncertainty deters investment in real options models. In the current model, it first deters investment, then, for intermediate levels of uncertainty, investment is hastened, and, finally, high levels of uncertainty deter investment again. This effect seems to be channeled through the threshold $X_{11}^{c}$ (Equations (14)). This is the threshold for investment in the first stage, in a staged investment strategy, after the issuance of the construction permit. The effect

\footnotetext{
${ }^{6}$ Our model converges to the Dixit and Pindyck (1994 ch. 10) sequential investment case, where investment occurs always in a single stage, when there is no competition, the exogenous event is imminent, and there is no discount $\left(\lambda_{E} \rightarrow \infty, \lambda_{C} \rightarrow 0\right.$, and $\left.\theta=1\right)$.
} 
Table 3: Numerical examples

\begin{tabular}{|c|c|c|c|c|c|c|c|c|}
\hline$\lambda_{E}$ & $\lambda_{C}$ & $\theta$ & $X_{1}$ & $X_{1}^{c}$ & $X_{2}$ & Type & Case & Eq. $X_{1}$ \\
\hline 0 & $\forall$ & $\forall$ & $\infty$ & - & 121.3 & - & - & - \\
\hline 0.05 & 0 & 0.9 & 151.7 & 145.5 & 121.3 & Single stage & $\overline{\mathrm{H}}$ & $(58)$ \\
\hline 0.05 & 0.008 & 0.9 & 124.0 & 123.7 & 121.3 & Single stage & $\mathrm{H}$ & $(58)$ \\
\hline 0.05 & 0.1 & 0.9 & 50.4 & 53.0 & 121.3 & Staged & A & (40) \\
\hline 0.05 & $\infty$ & 0.9 & 36.7 & 38.2 & 121.3 & Staged & A & $(40)$ \\
\hline 0.05 & 0 & 1 & 153.4 & 145.5 & 121.3 & Single stage & $\mathrm{H}$ & $(58)$ \\
\hline 0.05 & 0.01 & 1 & 121.9 & 116.7 & 121.3 & Staged & $\mathrm{D}$ & $(49)$ \\
\hline 0.05 & 0.1 & 1 & 54.5 & 53.0 & 121.3 & Staged & $\mathrm{C}$ & $(\overline{49})$ \\
\hline$\infty$ & 0 & 0.9 & 125.3 & 145.5 & 121.3 & Single stage & $\mathrm{F}$ & (53) \\
\hline$\infty$ & 0.008 & 0.9 & 96.7 & 123.7 & 121.3 & Single stage & $\mathrm{E}$ & 53 \\
\hline$\infty$ & 0.1 & 0.9 & 43.0 & 53.0 & 121.3 & Staged & A & $(40)$ \\
\hline$\infty$ & $\infty$ & 0.9 & 34.0 & 38.2 & 121.3 & Staged & A & 40 \\
\hline$\infty$ & 0 & 1 & 145.5 & 145.5 & 121.3 & Single stage & G & $(58)$ \\
\hline$\infty$ & 0.01 & 1 & 116.7 & 116.7 & 121.3 & Staged & $\mathrm{C}$ & 49 \\
\hline$\infty$ & 0.1 & 1 & 53.0 & 53.0 & 121.3 & Staged & $\mathrm{C}$ & $(49)$ \\
\hline
\end{tabular}

$\sigma=0.1, r=0.04, \alpha=0.02, K_{1}=10, K_{2}=50 . X_{1}$ is the stage 1 investment threshold. $X_{2}$ is the stage 2 investment threshold and it is obtained with Equation (6). $X_{1}^{c}$ is the staged 1 investment threshold after the permit is issued, and it is obtained with Equation (14) or 20), depending on the condition in Equation (15). Solving it for $\lambda_{C}$, the critical level is $\lambda_{C}^{*}=0.00928$. For $\lambda_{C}>\lambda_{C}^{*}$ the staged investment is the optimal strategy.

of uncertainty on $X_{11}^{c}$ is twofold: (i) on the one hand a higher uncertainty (lower $\eta_{1}$ ) increases the threshold and (ii) on the other hand it makes the option to invest in the development stage more valuable (increasing $a_{1} X^{\beta_{1}}$ ), which promotes investment. These two effects dominate for different levels of uncertainty. The figure also shows, as in the previous figure, that a discount in the investment cost can induce investment sooner before than after the permit is issued. This effect is higher for low levels of uncertainty. A high uncertainty decreases the incentive to secure the investment opportunity before the permit.

The effect of the investment costs are depicted in Figure 4. Higher investment costs delay investment. When the cost of the land $\left(K_{1}\right)$ is not sufficiently smaller than that of the development stage $\left(K_{2}\right)$, the firm invests in the first stage and waits for the permit, which will prompt the development stage investment.

\section{Conclusion}

This paper develops a model to determine the value and optimal timing of an opportunity to invest in a random start American real option. A random start American option materializes into an American option only after an exogenous event, such as a permit or a discovery, occurs. While waiting to invest the firm faces the risk of a hidden competitor 


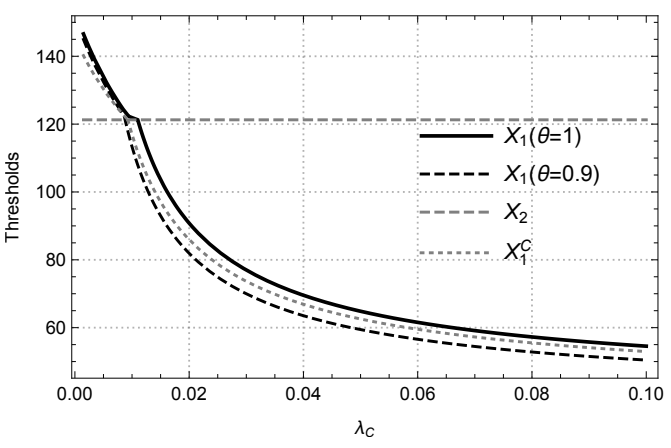

(a)

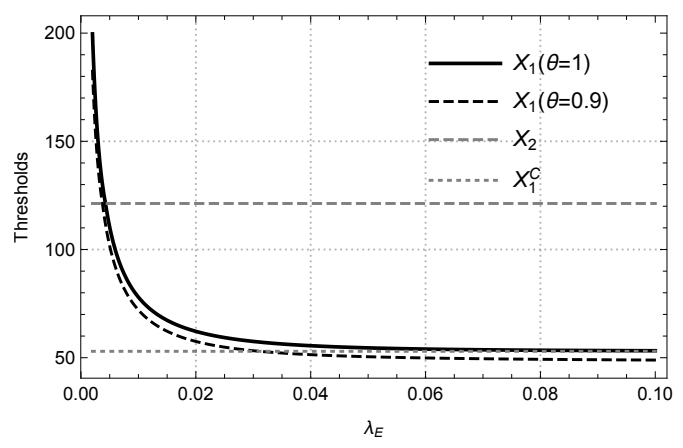

(b)

$\sigma=0.1, r=0.04, \alpha=0.02, \lambda_{C}=0.1, \lambda_{E}=0.05, K_{1}=10, K_{2}=50 . X_{1}$ is the stage 1 investment threshold and it is obtained with Equations 40, 49, 53 or 58, according to the cases presented in Table $1 . \quad X_{2}$ is the stage 2 investment threshold and it is obtained with Equation $(6) . X_{1}^{c}$ is the staged 1 investment threshold after the permit is issued,and it is obtained with Equation (14) or 20), depending on the condition in Equation 15 .

Figure 2: Sensitivity of the investment thresholds to $\lambda_{C}$ and $\lambda_{E}$

destroying the value of the opportunity to invest.

We show that investment is deterred when the risk of competition is low or the probability of arrival of a permission to invest in the development stage is also low. Investment is also deterred for high investment costs in both stages. The effect of uncertainty is shown to be non-monotonic. For low and high uncertainty levels an increase in uncertainty deters investment, and for intermediate uncertainty levels the effect is the reverse.

\section{References}

Armada, M. R., Kryzanowski, L., and Pereira, P. J. (2011). Optimal investment decisions for two positioned firms competing in a duopoly market with hidden competitors. European Financial Management, 17(2):305-330.

Armerin, F. (2017). Random start American perpetual options. $21^{\text {st }}$ Real Options Annual International Conference, Boston MA, USA.

Dixit, A. and Pindyck, R. (1994). Investment under uncertainty. Princeton University Press, New Jersey.

Lavrutich, M. N., Huisman, K. J., and Kort, P. M. (2016). Entry deterrence and hidden competition. Journal of Economic Dynamics and Control, 69:409-435.

Lint, O. and Pennings, E. (1998). R\&D as an option on market introduction. RED Management, 28(4):279-287. 


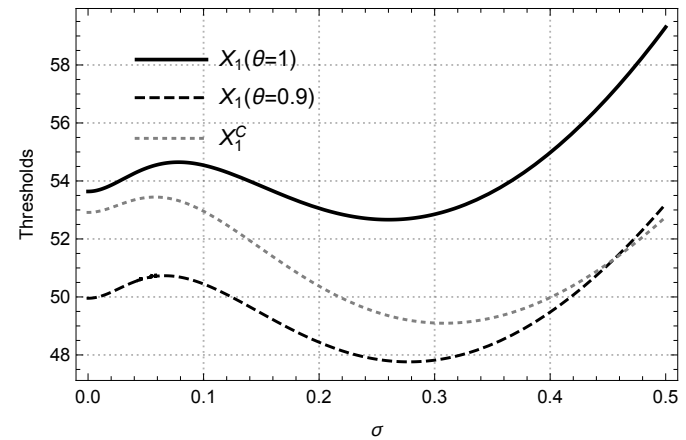

$\sigma=0.1, r=0.04, \alpha=0.02, \lambda_{C}=0.1, \lambda_{E}=0.05, K_{1}=10, K_{2}=50 . X_{1}$ is the stage 1 investment threshold and it is obtained with Equations (40), (49), (53) or (58), according to the cases presented in Table $1 \quad X_{2}$ is the stage 2 investment threshold and it is obtained with Equation (6). $X_{1}^{c}$ is the staged 1 investment threshold after the permit is issued,and it is obtained with Equation (14) or (20), depending on the condition in Equation (15).

Figure 3: Sensitivity of the investment thresholds to $\sigma$

McDonald, R. and Siegel, D. (1986). The value of waiting to invest. The Quarterly Journal of Economics, 101(4):707-727.

Pereira, P. J. and Armada, M. R. (2013). Investment decisions under hidden competition. Economics Letters, 121(2):228-231. 


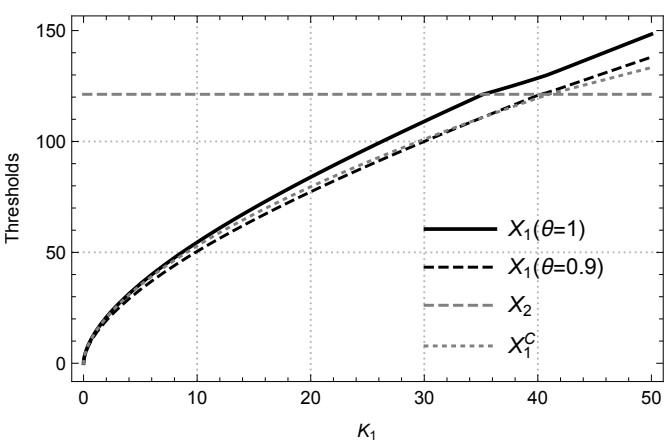

(a)

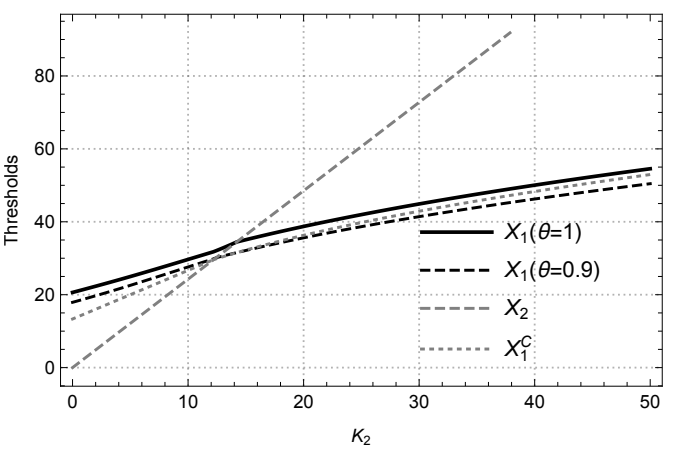

(b)

$\sigma=0.1, r=0.04, \alpha=0.02, \lambda_{C}=0.1, \lambda_{E}=0.05, K_{1}=10, K_{2}=50 . X_{1}$ is the stage 1 investment threshold and it is obtained with Equations 40, 49, ,53 or 58, according to the cases presented in Table 1 . $X_{2}$ is the stage 2 investment threshold and it is obtained with Equation (6). $X_{1}^{c}$ is the staged 1 investment threshold after the permit is issued,and it is obtained with Equation (14) or (20), depending on the condition in Equation (15).

Figure 4: Sensitivity of the investment thresholds to $K_{1}$ and $K_{2}$ 